\title{
Variation of the default mode network with altered alertness levels induced by propofol
}

This article was published in the following Dove Press journal:

Neuropsychiatric Disease and Treatment

7 October 2015

Number of times this article has been viewed

\author{
Xiaoyuan Liu' \\ Huandong $\mathrm{Li}^{2}$ \\ Fang Luo' \\ Lei Zhang ${ }^{3}$ \\ Ruquan Han' \\ Baoguo Wang ${ }^{4}$ \\ 'Department of Anesthesiology, \\ Beijing Tiantan Hospital, Capital \\ Medical University, ${ }^{2}$ Brainnetome \\ Center, Institute of Automation, \\ Chinese Academy of Sciences, \\ ${ }^{3}$ Department of Neuroradiology, \\ Beijing Neurosurgical Institute, \\ ${ }^{4}$ Department of Anesthesiology, \\ Sanbo Brain Hospital, Capital Medical \\ University, Beijing, People's Republic \\ of China
}

Background: The default mode network (DMN) is closely associated with the maintenance of alertness and cognitive functions. This study aimed to observe the changes in DMN induced by increasing doses of propofol and progressively deepening sedation.

Methods: Twelve healthy subjects were selected; they received target-controlled infusion of propofol (1.0 and $3.0 \mu \mathrm{g} / \mathrm{mL}$ of plasma) and underwent functional magnetic resonance imaging before sedation and when they achieved light and deep sedation states. The average degree, average shortest path length, global efficiency, local efficiency, and clustering coefficient of DMN were assessed to study the overall and internal changes of DMN with gradual changes in alertness level, as well as the relationship between thalamus and DMN. Meanwhile, basic vital signs and respiratory inhibition were recorded.

Results: DMN parameters were gradually inhibited with decreasing level of alertness, the differences were significant between light sedation and awake states (all $P<0.01$ ), but not between deep and light sedation states. However, the shortest path lengths of the posterior cingulate cortex, medial prefrontal cortex, and lateral parietal cortexes in the DMN were significantly increased under deep sedation.

Conclusion: Overall, DMN is propofol-sensitive. A small dose of propofol can significantly inhibit the DMN, affecting the level of alertness. The posterior cingulate cortex, medial prefrontal cortex, and lateral parietal cortexes in the DMN are less sensitive to propofol, and could be significantly inhibited by a higher concentration of propofol, further reducing the level of alertness.

Keywords: default mode network, propofol, functional connectivity, sedation, anesthesia

\section{Introduction}

With the development of functional magnetic resonance imaging (fMRI), it is widely accepted that any simple activity or functional and status changes in the brain originate from complex interactions of numerous brain regions and brain networks, rather than depending on a single brain region. ${ }^{1}$ In 2001 , Raichle et $\mathrm{al}^{2}$ first suggested that there is a highly ordered functional network in the brain in awake and resting states with the absence of focus on the outside world, namely, the default mode network (DMN). Since then, many studies have confirmed the existence of the DMN and have pointed out that it is closely associated with maintenance of alertness ${ }^{3,4}$ including monitoring the external environment, maintaining self-consciousness, ${ }^{5}$ and emotional processing. ${ }^{6}$ The DMN undergoes significant changes during physiological sleep ${ }^{3}$ and in conditions involving cognitive impairment such as Alzheimer's disease ${ }^{7}$ and Parkinson's disease. ${ }^{8}$ Given the important role of DMN in cognition and alertness, many scholars have started to investigate the relationship between altered alertness and anesthetics. ${ }^{9-11}$
Correspondence: Baoguo Wang Department of Anesthesiology, Sanbo Brain Hospital, Capital Medical University, Number 50, Xiang Shan Yi-Ke-Song, Haidian District, Beijing 100093, People's Republic of China

$\mathrm{Tel}+86 \quad 13621278793$

Fax +86 2I 64085875

Email wangbg605@sina.com 
Propofol is the most widely used sedative-hypnotic drug in clinical anesthesia. However, current studies of the effects of propofol on DMN have failed to provide detailed observation and comprehensive investigation of overall and internal changes and effects in the DMN with the gradual changes of alertness. ${ }^{12,13}$ This study aimed to investigate the DMN and its changes with increasing doses of propofol and progressive deepening of sedation.

\section{Methods}

\section{Participants}

This study was performed in the Beijing Tiantan Hospital (Beijing, People's Republic of China), and all the participants were workers responsible for cleaning the hospital. Inclusion criteria were: 1) aged 18-40 years; 2) right-handed; 3) no comorbidities; 4) American Society of Anesthesiologists grade I; and 5) body mass index $18.5-25 \mathrm{~kg} / \mathrm{m}^{2}$. Exclusion criteria were: 1) history of any chronic, mental, or nervous system disease; 2) history of traumatic brain injury or surgery; 3) history of long-term administration of sedative or analgesic drugs; 4) history of long-term heavy smoking or drinking; or 5) magnetic resonance imaging (MRI) contraindications.

The basic procedures and requirements of the experiments were explained to the participants, and they signed written informed consent forms. On the day of experiment, the explanations were provided again. Participants were allowed to exit the experiment for any reason during the experimental process. The study was approved by the Ethics Committee of Beijing Tiantan Hospital, Capital Medical University. The whole experimental process accorded with the Declaration of Helsinki.

\section{Experimental procedures}

\section{Propofol administration}

Studies have shown that a $2.3-3.5 \mu \mathrm{g} / \mathrm{mL}$ dose of propofol may lead to a loss of alertness in $50 \%$ of individuals. ${ }^{14}$ Therefore, concentrations of $1.0,1.5,2.0,2.5$, and $3.0 \mu \mathrm{g} / \mathrm{mL}$ were pretested. Concentrations of $1.0 \mu \mathrm{g} / \mathrm{mL}$ achieved an Observer's Assessment of Alertness/Sedation scale (OAA/S) score close to 4 (lethargic response to name spoken in normal tone), and $3.0 \mu \mathrm{g} / \mathrm{mL}$ achieved an OAA/S score of 2-3 (respond only after name is called loudly and/or repeatedly and/or after mild shaking). ${ }^{15}$

\section{MRI}

Participants fasted and avoided drinking 6 hours and 4 hours prior to the experiments, respectively. Half an hour before scanning, a catheter was placed in the participants' left median cubital vein, and 200-300 mL of lactated Ringer's solution was infused. Participants' heads were fixed in the coil, and the skull was immobilized with sponge pads to prevent head movements. Rubber earplugs were used to reduce noise. Scanning was started after a 10-minute rest. Participants were required to close their eyes, to maintain calm breathing, and to keep still without focused thinking. When the experiments started, the infusion was maintained, and participants received nasal oxygen through a catheter. A MRI monitor (GE Healthcare Bio-Sciences Corp., Piscataway, NJ, USA) was used for real-time electrocardiographic monitoring, noninvasive blood pressure (mean arterial pressure, right upper arm), oxygen saturation, and end-tidal carbon dioxide pressure. The OAA/S and respiratory rate were recorded, and the presence of respiratory depression (oxygen saturation $<95 \%$; respiratory rate $<10$ breaths/min; apnea time $>6$ seconds) was observed. An AESTIVA MRI anesthesia machine (GE Healthcare Bio-Sciences Corp.) was used as backup.

Target-controlled infusion (MODULE DPS ORCHESTRA IS3; Fresenius Kabi Pharmaceutical Co., Ltd., Baden Humboldt, Germany) of propofol (batch number FY707; AstraZeneca plc, London, UK) was immediately performed after fMRI (maximum $500 \mathrm{~mL} / \mathrm{h}$ to maintain a plasma concentration of $1.0 \mu \mathrm{g} / \mathrm{mL})$. OAA $/ \mathrm{S}$ was assessed after the target concentration had been reached for 10 minutes. The second blood oxygen level-dependent scan was conducted while maintaining propofol infusion. Plasma concentration was increased to $3.0 \mu \mathrm{g} / \mathrm{mL}$ and the same examinations were performed.

\section{Data acquisition}

MRI was acquired on a 3.0 T MRI system (Magnetom Trio; Siemens, Erlangen, Germany). fMRI data were acquired using an echo-planar imaging sequence (repetition time $/$ echo time $=2,000 / 30 \mathrm{~ms}$; flip angle $=90^{\circ}$; field of view $=256 \times 256 \mathrm{~mm}^{2}$; matrix $=64 \times 64$; slice thickness $/$ gap $=4.0 / 0.0 \mathrm{~mm}$; voxel size $=4.0 \times 4.0 \times 4.0 \mathrm{~mm}^{3} ; 32$ interleaved slices). Each session lasted 5 minutes and 150 volumes were obtained. Additionally, high-resolution T1-weighted structural images were acquired at $1 \mathrm{~mm}$ isotropic resolution in the sagittal plane.

\section{Data preprocessing}

fMRI data were preprocessed using SPM8 (http://www.fil. ion.ucl.ac.uk/spm) and Data Processing Assistant for RestingState fMRI. ${ }^{16}$ The following preprocessing steps were performed: discarding the first five slices for magnetization 
equilibrium; slice timing correction; head motion correction; spatial normalization; resampling to $3 \times 3 \times 3 \mathrm{~mm}$ size; smoothing with a $6 \mathrm{~mm}$ full-width at half-maximum Gaussian kernel; regressing nuisance signals (six motion parameters, global mean signal, and average blood oxygen level-dependent signals in ventricular and white matter regions); and temporal filtering (0.01-0.1 Hz). Two participants were excluded because of a displacement $>2 \mathrm{~mm}$, or a spin $>2^{\circ}$.

\section{Region definition}

We used regions of interest (ROIs) to define the DMN according to previous studies (Table 1). ${ }^{17,18}$ All ROIs were defined as $12 \mathrm{~mm}$ spherical regions centered on the coordinates of a priori ROI. A thalamus ROI was defined to investigate the interaction between thalamus and DMN. Thalamus seed was extracted from the Harvard-Oxford cortical structural atlas (http://fsl.fmrib.ox.ac.uk/fsl/fslwiki/Atlases) with a threshold of $50 \%$ minimum probability.

\section{Functional connectivity}

Regional time series were extracted by averaging the fMRI time series of all voxels within the ROI. Pearson's correlation coefficient were computed between each pair of regional time series. The resulting correlation was transformed to approximate Gaussian distribution using Fisher's $r-z$ transformation. Thus, we obtained a $12 \times 12 \mathrm{DMN}$ functional connectivity matrix for each subject.

\section{Graph-theory analysis}

Graph-theory analysis was run on adjacency matrix $\mathrm{G}$, defined by an undirected, weighted, and simple graph $G=(V, E)$, where $\mathrm{V}=\{1 \ldots, \mathrm{N}\}$ represents the node set and $\mathrm{E}=\{\mathrm{e}(\mathrm{i}, \mathrm{j})\}$ is the edge set. The e $(i, j)$ is the edge between nodes $i$ and $j$ $(\mathrm{i}, \mathrm{j} \in \mathrm{V})$ and is associated with functional connectivity weight $\mathrm{w}_{\mathrm{ij}}\left(0 \leq \mathrm{w}_{\mathrm{ij}} \leq 1\right)$. A 0.1 threshold was applied to remove weak correlations caused by signal noise:

$$
\begin{gathered}
e(i, j)=e_{i j}, w_{i j} \geq 0.1 \\
e_{i j}=\left\{\begin{array}{l}
0, r_{i j}<0.1 \\
r_{i j}, r_{i j} \geq 0.1
\end{array}\right.
\end{gathered}
$$

where $r_{i j}$ is the Pearson's correlation coefficient between $\mathrm{i}$ and $\mathrm{j}$. The degree of each node, $\mathrm{K}_{\mathrm{i}, \mathrm{i} \in \mathrm{v}}$, was defined as the sum weights of connections linked to node $i$ :

$$
K_{\mathrm{i}}=\sum_{j \in v} w_{i j}
$$

The degree $\mathrm{K}$ of a graph was the average degree of all the nodes in the graph:

$$
K=\frac{1}{N} \sum_{i \in G} K_{i}
$$

which is a measure to evaluate the strength of the brain functional network.

The clustering coefficient $\mathrm{C}_{\mathrm{i}}$ can quantify how close the neighbors of the node $\mathrm{i}$ are. There are several definitions for clustering coefficient in weighted graphs. Here, we used the weighted clustering coefficient defined by Onnela et $\mathrm{al}^{19}$ which was previously implemented for functional brain network analysis: ${ }^{20}$

$$
C_{i}=\frac{1}{k_{i}\left(k_{i}-1\right)} \sum_{j, h \neq i}\left(w_{i j} w_{i h} w_{j h}\right)^{1 / 3}
$$

The clustering coefficient of the network $\mathrm{C}$ was the average of the clustering coefficient of all nodes and is a measure

\begin{tabular}{|c|c|c|c|}
\hline Brain region & Abbreviations & MNI coordinates & BA \\
\hline Anterioredial prefrontal cortex & aMPFC & $-3,57,21$ & 10 \\
\hline Left superior frontal cortex & L.Sup.F & $-12,45,48$ & 8 and 9 \\
\hline Right superior frontal cortex & R.Sup.F & $21,42,48$ & 8 and 9 \\
\hline Medial prefrontal cortex (ventral) & vMPFC & $-3,39,-2$ & 10 \\
\hline Left inferior temporal cortex & L.IT & $-54,-3,-30$ & 20 and 21 \\
\hline Right inferior temporal cortex & R.IT & $54,0,-30$ & 20 and 21 \\
\hline Left parahippocampal gyrus & L.PHC & $-24,-18,-27$ & 35 and 36 \\
\hline Right parahippocampal gyrus & R.PHC & $24,-12,-27$ & 35 and 36 \\
\hline Posterior cingulate cortex & PCC & $-3,-45,33$ & 31 \\
\hline Left lateral parietal cortex & L.LatP & $-54,-69,36$ & 39 and 40 \\
\hline Right lateral parietal cortex & R.LatP & $54,-63,33$ & 39 and 40 \\
\hline Cerebellar tonsils & Cereb & $9,-5 \mathrm{I},-45$ & NA \\
\hline
\end{tabular}
of functional segregation:

$$
C=\frac{1}{N} \sum_{i \in G} C_{i}
$$

Table I Seed regions for the default mode network

Abbreviations: BA, Brodmann area; MNI, Montreal Neurological Institute; NA, not applicable. 
The shortest path length of two nodes $\mathrm{L}_{\mathrm{ij} \in \mathrm{v}}$, refers to the length of the shortest path between node $i$ and $j$. The shortest path length of each node was defined as the mean shortest path length between node $\mathrm{i}$ and other nodes:

$$
L_{i}=\frac{1}{n-1} \sum_{j \in G, j \neq i} L_{i j}
$$

where

$$
d_{i j}=\sum_{\beta h k \in p_{i \rightarrow j}} l_{h k}
$$

is the shortest weighted path length between $\mathrm{i}$ and $\mathrm{j}$, and we used an inverse to map weight to length:

$$
l_{h k}=\frac{1}{w_{h k}}
$$

and $p_{i \rightarrow j}$ is the shortest weighted path from $i$ to $j$. The characteristic path length of the network $L$ is the average of the shortest path lengths between the nodes:

$$
L=\frac{1}{N} \sum_{i \in G} L_{i}
$$

$\mathrm{L}$ is a basic method for measuring integration of the network.

The global efficiency of the network is defined by the inverse of the harmonic mean of the shortest path length between each pair of nodes:

$$
E=\frac{1}{n} \sum_{i \in G} E_{i}=\frac{1}{n} \sum_{i \in G} \frac{\sum_{i \in G, j \neq i} d_{i j}^{-1}}{n-1}
$$

where $E_{i}$ is the efficiency of node $i$.

The local efficiency of the network is a measure of the fault tolerance of the network:

$$
E_{l o c}=\frac{1}{n} \sum_{i \in G} \mathrm{E}_{l o c, i}=\frac{1}{n} \sum_{i \in G} \frac{\sum_{j, h \in G, j \neq i\left(W_{i j} W_{i h}\left[d_{j h}\left(N_{i}\right)\right]^{-1}\right)^{1 / 3}}}{n-1}
$$

where $\mathrm{E}_{\text {loc, } \mathrm{i}}$ is the local efficiency of node $\mathrm{i}$, and $\mathrm{d}_{\mathrm{jh}}\left(\mathrm{N}_{\mathrm{i}}\right)$ is the shortest path length between $\mathrm{j}$ and $\mathrm{h}$, which contains only neighbors of $\mathrm{i}$, using a 0.1 threshold.

\section{Statistical analysis}

Statistical analysis focused on differences in functional connectivity and graph measures between different states of sedation. We used analysis of repeated measure analysis of variance with the Student's post hoc $t$-test in MATLAB (version 2012a; MathWorks, Natick, MA, USA) for all statistical operations. $P<0.01$ was defined as significantly different.

\section{Results}

\section{General information}

Fourteen healthy Asian participants were enrolled (eight men and six women). Two participants were excluded due to head movements. Twelve participants were included (seven men and five women), aged 18-39 years (mean of 25.9 years), and weighing 48-75 kg (mean of $58 \mathrm{~kg}$ ). Physiological parameters at each stage are displayed in Table 2. Their $\mathrm{OAA} / \mathrm{S}$ scores gradually decreased with increasing dose of propofol (Table 3).

\section{Changes in the overall DMN}

Compared with the awake state, all parameters (mean degree, shortest path length, global efficiency, local efficiency, and clustering coefficient) displayed significant changes in the light and deep sedation states, while there was no difference between light and deep sedation states (Table 4).

\section{Variation of each DMN node}

Compared with the awake state, the shortest path lengths of the left inferior temporal cortex (L.IT) $(2.48 \pm 0.49$ to $3.25 \pm 0.77)$, right inferior temporal cortex (R.IT) $(2.63 \pm 0.78$ to $3.30 \pm 0.73$ ), right superior frontal cortex (R.Sup.F) $(2.22 \pm 0.34$ to $2.94 \pm 0.80)$, right parahippocampal gyrus $(3.41 \pm 0.63$ to $4.48 \pm 1.00)$, and left lateral parietal cortex (L.LatP) (2.26 \pm 0.39 to $2.89 \pm 0.62)$ in the light sedation state were significantly increased. The shortest path lengths of the posterior cingulate cortex (PCC) (3.04 \pm 0.80 to $3.80 \pm 0.82)$, anterior medial prefrontal cortex (aMPFC) (3.02 \pm 0.91 to $3.91 \pm 1.04)$, L.LatP ( $2.89 \pm 0.62$ to $3.95 \pm 0.94)$, and right lateral parietal cortex (R.LatP) (3.21 \pm 0.84 to $4.09 \pm 0.81)$ were

Table 2 Physiological parameters at the awake, light sedation, and deep sedation states

\begin{tabular}{llll}
\hline Index & Awake state & $\begin{array}{l}\text { Light sedation state, plasma } \\
\text { concentration of } 1.0 \mu \mathbf{g} / \mathbf{m L}\end{array}$ & $\begin{array}{l}\text { Deep sedation state, plasma } \\
\text { concentration of } 3.0 \mu \mathrm{g} / \mathbf{m L}\end{array}$ \\
\hline $\mathrm{MAP}$ & $88 \pm 8$ & $82 \pm 8$ & $75 \pm 7$ \\
$\mathrm{HR}$ & $66 \pm 9$ & $64 \pm 7$ & $68 \pm 8$ \\
$\mathrm{SPO}_{2}$ & $100 \pm 0$ & $99 \pm 1$ & $98 \pm 2$ \\
$\mathrm{EtCO}_{2}$ & $38.1 \pm 2.4$ & $38.9 \pm 2.1$ & $44.3 \pm 2.7$ \\
\hline
\end{tabular}

Note: Results are presented as mean \pm standard deviation.

Abbreviations: $\mathrm{EtCO}_{2}$, end-tidal carbon dioxide; $\mathrm{HR}$, heart rate; MAP, mean arterial pressure; $\mathrm{SpO}_{2}$, oxygen saturation. 
Table 3 Number of participants in three different alertness states with Observer's Assessment of Alertness/Sedation scale (OAA/S) score

\begin{tabular}{|c|c|c|c|}
\hline OAA/S score & Awake state & $\begin{array}{l}\text { Light sedation } \\
\text { state }\end{array}$ & $\begin{array}{l}\text { Deep sedation } \\
\text { state }\end{array}$ \\
\hline 5 & 12 & 1 & 0 \\
\hline 4 & 0 & 11 & 0 \\
\hline 3 & 0 & 0 & 3 \\
\hline 2 & 0 & 0 & 9 \\
\hline
\end{tabular}

significantly increased in the deep sedation state compared with light sedation (Figure 1).

\section{Variations of functional connectivity}

Compared with the awake state, the connectivity between some pairs of nodes (R.IT and R.Sup.F, R.LatP, or cerebella tonsils, respectively; aMPFC and L.LatP; and L.IT and right parahippocampal gyrus) were significantly attenuated (Figures 2 and $3 \mathrm{~A}$ ) under light sedation. Compared with the light sedation state, the connectivity between some pairs of nodes (cerebella tonsils and L.LatP, R.LatP, R.Sup.F, or aMPFC, respectively; L.LatP and left superior frontal cortex; L.IT and aMPFC; and R.Sup.F and R.IT) were significantly attenuated under deep sedation (Figures 2 and 3B).

In addition, with decreasing alertness, the functional connectivity between thalamus and R.Sup.F decreased $(P<0.01$ awake vs light sedation).

\section{Discussion}

In the present study, DMN-related parameters (degree, average shortest path length, global efficiency, local efficiency, and clustering coefficient) reflected the activity of the overall network through the internal information integration ability of the network, information processing efficiency, and degree and extent of connectivity. ${ }^{21}$ Our results suggested that the DMN was progressively inhibited with propofol in a dosedependent manner. The connectivity among nodes in the

Table 4 DMN network attribute parameters in three different alertness states

\begin{tabular}{|c|c|c|c|}
\hline Index & $\begin{array}{l}\text { Awake } \\
\text { state }\end{array}$ & $\begin{array}{l}\text { Light } \\
\text { sedation } \\
\text { state }\end{array}$ & $\begin{array}{l}\text { Deep } \\
\text { sedation } \\
\text { state }\end{array}$ \\
\hline Mean degree & $4.58 \pm 0.7$ I & $3.66 \pm 1.0 I^{\Delta}$ & $2.97 \pm 0.78 *$ \\
\hline Shortest path length & $2.74 \pm 0.4 I$ & $3.46 \pm 0.83^{\Delta}$ & $4.31 \pm 0.91 *$ \\
\hline Global efficiency & $0.45 \pm 0.05$ & $0.37 \pm 0.08^{\wedge}$ & $0.31 \pm 0.06 *$ \\
\hline Local efficiency & $0.39 \pm 0.06$ & $0.30 \pm 0.09^{\Delta}$ & $0.24 \pm 0.07^{*}$ \\
\hline Clustering coefficient & $0.38 \pm 0.07$ & $0.29 \pm 0.094$ & $0.23 \pm 0.07 *$ \\
\hline
\end{tabular}

Notes: Results are shown as mean \pm standard deviation, $n=\mid 2$. $P<0.01$ between the three groups for all variables, according to analysis of variance. $\Delta P<0.01$ between light sedation and awake states; $* P<0.0$ l between deep sedation and awake states. There was no significant difference between light and deep sedation states. Abbreviation: DMN, default mode network.
DMN gradually decreased with increasing dose of propofol. These results were consistent with the conclusion that the DMN was suppressed using sevoflurane ${ }^{9}$ and propofol. ${ }^{10,22}$

In the present study, the changes of overall and functional connectivity among internal nodes in the DMN were consistent, suggesting that propofol gradually reduced the level of alertness and inhibited overall DMN. Thus, it can be inferred that an increasing dose of propofol was able to gradually reduce network activity through comprehensively inhibiting the integration and processing of the overall DMN information and connectivity among DMN's internal nodes, leading to a progressive decrease in alertness.

Some other studies have investigated the effects of different concentrations of propofol on DMN, but they did not describe the specific changes of DMN in light and deep sedation states. ${ }^{10,22} \mathrm{We}$ found that all DMN parameters displayed significant changes in the light sedation state, while they only showed slight additional changes when deepening the sedation state. Therefore, we believe that the DMN can be considered an effective indicator of the impact of propofol on alertness. We speculate that this is one of the mechanisms by which a low dose of propofol is able to change the level of alertness.

In this study, PCC, aMPFC, and bilateral LatP were the nodes with the highest activity. Compared with overall DMN, they were suppressed only with a higher concentration of propofol due to their lower sensitivity, and their inhibition was related to further decreasing levels of alertness.

PCC is a core node in DMN, ${ }^{23,24}$ which has extensive fiber projection and transmission with $\mathrm{PFC}$, superior frontal cortex, LatP, and parahippocampal gyrus (PHC); thus, PCC becomes a hub of information processing within the DMN. ${ }^{24,25,26}$ Greicius et al found that the PCC functional connectivity in the DMN was significantly reduced with midazolam. ${ }^{27} \mathrm{Sta}-$ matakis et al found that the functional connectivity of PCC in the DMN was retained under light sedation with propofol doses of $0.27 \mu \mathrm{g} / \mathrm{mL}$ and $0.67 \mu \mathrm{g} / \mathrm{mL} .{ }^{11}$

aMPFC is an important node in DMN, involved in integrating internal and external environments, emotions, and other information, and in information extraction. ${ }^{28}$ aMPFC and PCC are correlated with spontaneous thinking activities, ${ }^{29}$ including memories and vision of the future. ${ }^{30,31}$ Patients with medial prefrontal cortex impairment may have apparent deficiency of spontaneous thinking. ${ }^{32}$ Some studies have pointed out that PCC and aMPFC display reduced activity or attenuated functional connectivity in deep sleep, ${ }^{3}$ as well as with cognitive dysfunction caused by diseases such as Parkinson's disease ${ }^{33}$ and Alzheimer's disease. ${ }^{7}$ LatP is anatomically close to PCC and they are considered as a whole in some studies. ${ }^{7}$ 


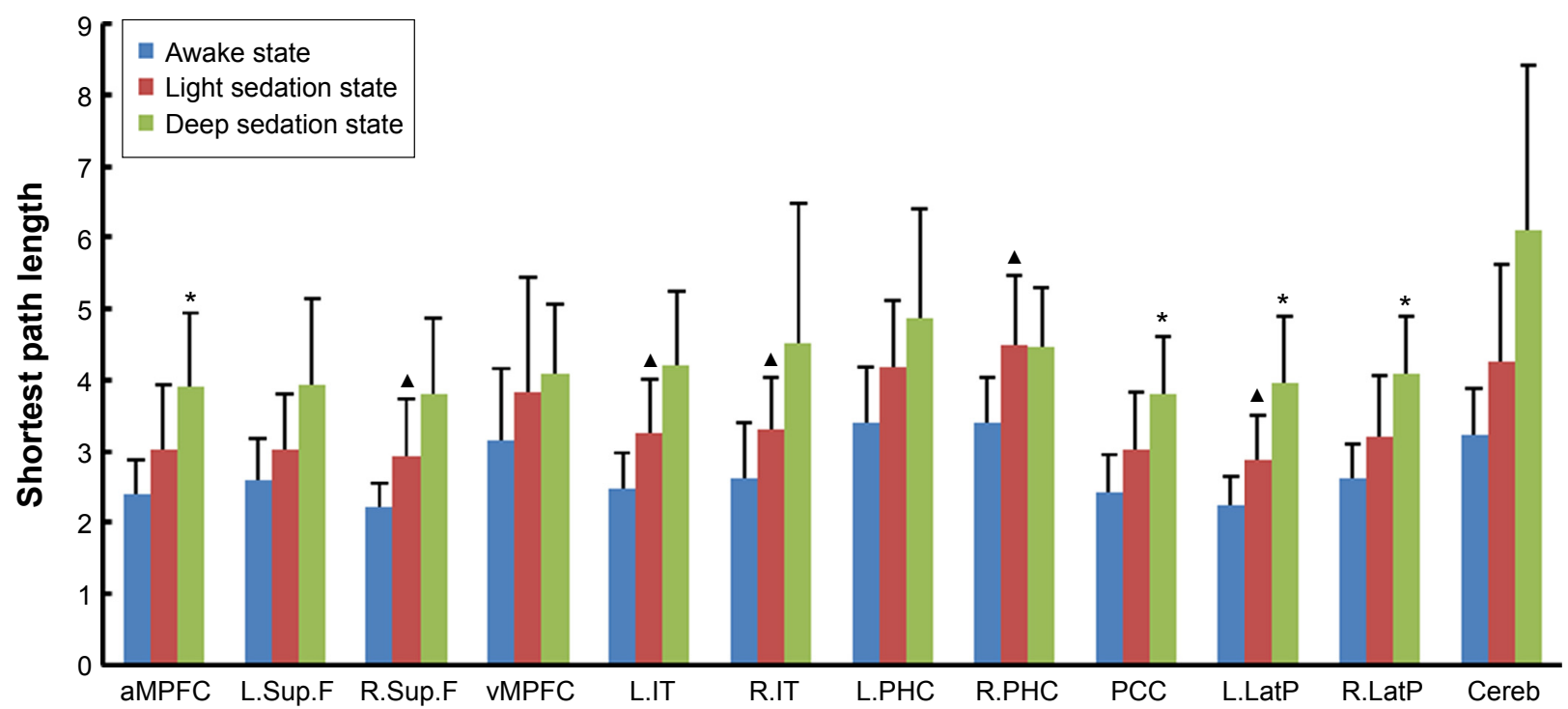

Figure I Variation of shortest path length of DMN nodes under different states of alertness.

Notes: The bars indicate mean \pm standard deviation. ${ }^{\Delta}$ Light sedation state vs awake state $P<0.0$ I. *Deep sedation state vs light sedation state $P<0.0$ I.

Abbreviations: aMPFC, anterior medial prefrontal cortex; Cereb, cerebellar tonsils; DMN, default mode network; L.IT, left inferior temporal cortex; L.LatP, left lateral parietal cortex; L.PHC, left parahippocampal gyrus; L.Sup.F, left superior frontal cortex; PCC, posterior cingulate cortex; R.IT, right inferior temporal cortex; R.LatP, right lateral parietal cortex; R.PHC, right parahippocampal gyrus; R.Sup.F, right superior frontal cortex; vMPFC, medial prefrontal cortex (ventral).

Based on this, we speculated that PCC, aMPFC, and bilateral LatP are high metabolic nodes in DMN at rest, playing a pivotal role in the network. Compared with the overall DMN, they are less sensitive to propofol, and their inhibition contributes to further decrease of the level of alertness. Thus, they are core sites for achieving deep sedation.

In this study, bilateral hemispheres in the same brain regions retained strong functional connectivity under deep sedation. Recent studies investigated the actual significance of the changes in functional connectivity between bilateral cortexes, but knowledge is still lacking. The activity synchronization of bilateral superior frontal cortex was significantly correlated with the accuracy of performance and level of alertness. ${ }^{34,35}$ Ono et al found that participants who had established a good functional connectivity between bilateral premotor cortexes were better at memory tasks. ${ }^{36}$

In this study, the shortest path lengths of the bilateral temporal lobes were significantly increased from awake to light sedation states, while the functional connectivity between the temporal lobe and multiple nodes were significantly
A

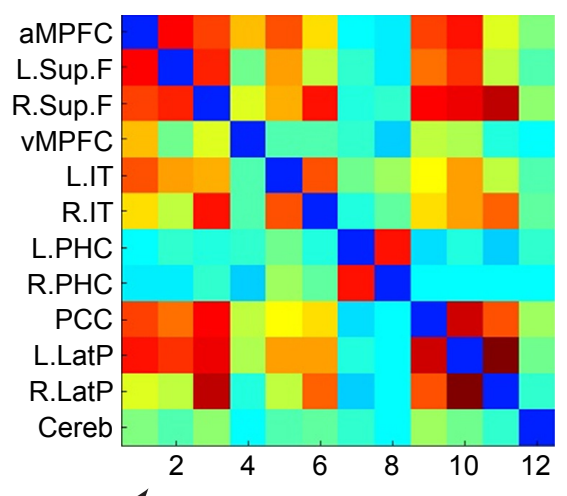

B

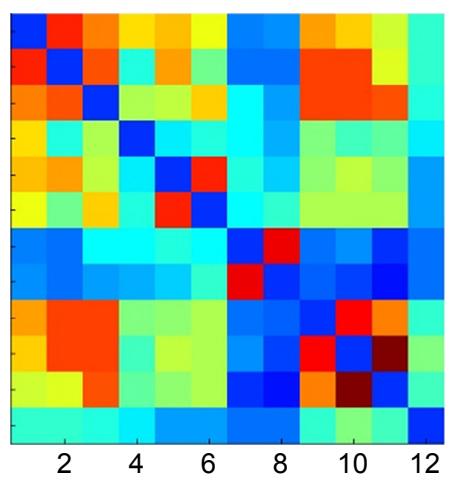

C

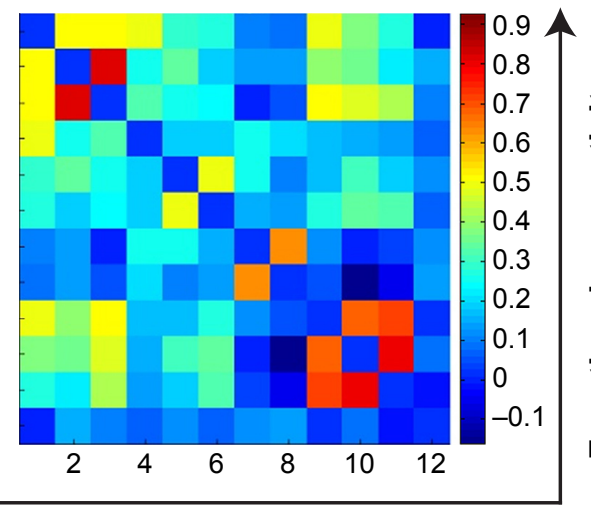

\section{Brain region in DMN}

Figure 2 Mean functional connectivity within DMN for awake (A), light sedation (B), and deep sedation (C) states.

Notes: $X$ coordinates represent each brain region in DMN, where I-12 represents aMPFC, L.Sup.F, R.Sup.F, vMPFC, L.IT, R.IT, L.PHC, R.PHC, PCC, L.LatP, R.LatP, and Cereb, respectively, and the color bar on the right represents the strength of functional connectivity.

Abbreviations: aMPFC, anterior medial prefrontal cortex; Cereb, cerebellar tonsils; DMN, default mode network; L.IT, left inferior temporal cortex; L.LatP, left lateral parietal cortex; L.PHC, left parahippocampal gyrus; L.Sup.F, left superior frontal cortex; PCC, posterior cingulate cortex; R.IT, right inferior temporal cortex; R.LatP, right lateral parietal cortex; R.PHC, right parahippocampal gyrus; R.Sup.F, right superior frontal cortex; vMPFC, medial prefrontal cortex (ventral). 

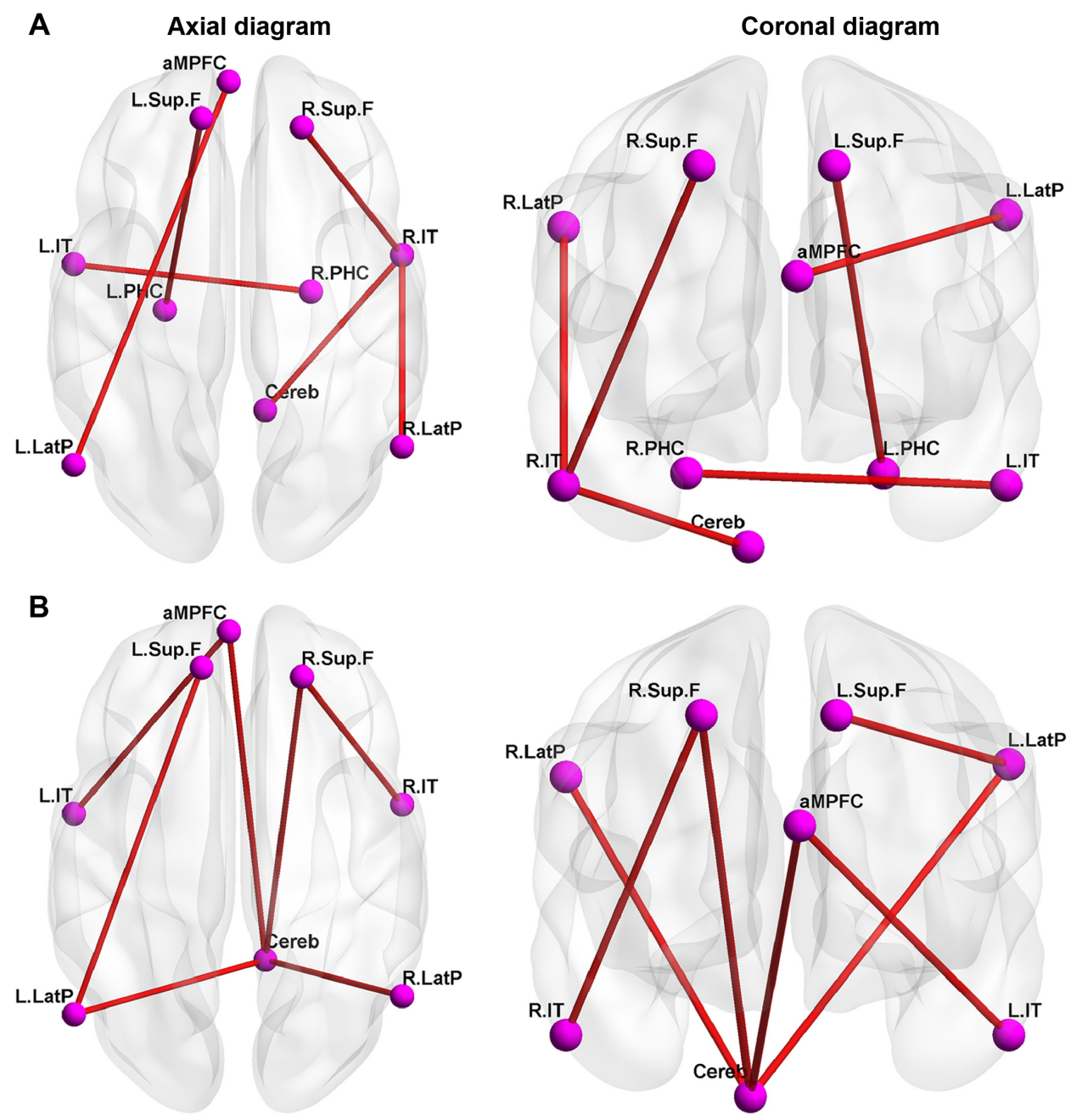

Figure 3 Significantly reduced functional connectivity within DMN from awake state to light sedation state and light sedation state to deep sedation state.

Notes: (A) Significantly reduced functional connectivity within DMN from awake state to light sedation state $(P<0.01)$. (B) Significantly reduced functional connectivity within DMN from light sedation state to deep sedation state $(P<0.0 \mathrm{I})$.

Abbreviations: aMPFC, anterior medial prefrontal cortex; Cereb, cerebellar tonsils; DMN, default mode network; L.IT, left inferior temporal cortex; L.LatP, left lateral parietal cortex; L.PHC, left parahippocampal gyrus; L.Sup.F, left superior frontal cortex; R.IT, right inferior temporal cortex; R.LatP, right lateral parietal cortex; R.PHC, right parahippocampal gyrus; R.Sup.F, right superior frontal cortex.

attenuated, suggesting that signal processing efficiency was greatly decreased, and its connectivity with other nodes in the network had significantly declined. Although participants were wearing earplugs to minimize noise, noise was not completely blocked. Therefore, it was equivalent to applying sound stimuli in the experiments. Some studies pointed out that although the response of auditory cortex to sound stimuli was reduced in different degrees of sedation with propofol, it was still present. ${ }^{37}$ Plourde et al found that the response of auditory cortex to sound stimuli was not completely blocked when alertness disappeared with a large dose of propofol, but the specific responses to different sounds and vocabulary stimuli were lost. ${ }^{38}$ The level of alertness depends on the effective exchange and integration of information between cortexes. ${ }^{39}$ More importantly, the functional connectivity of the temporal lobe as the auditory cortex with multiple nodes in the DMN was significantly decreased, suggesting that the information integration was greatly reduced, leading to a loss of significance of the sound stimuli. Thus, inhibition of DMN is part of the mechanism of decreased hearing in early sedation using propofol.

In the present study, there was no association between the thalamus and changes in DMN. Therefore, we speculated that they might play different roles in the occurrence, maintenance, and changes of alertness. Early studies of the mechanisms of altered alertness suggested that the thalamus was the main area of interest due to its specific anatomic status. However, the close association between alertness and the brain network is now recognized. ${ }^{12,13}$ Indeed, compared with the awake state, the functional connectivity of the thalamus and DMN was significantly changed in unconsciousness, ${ }^{12,22}$ while the relationship between the thalamus and DMN did not change significantly under light 
sedation. ${ }^{13}$ The present study further showed that compared with the awake state, the correlation between the thalamus and DMN did not show significant changes under light and deep sedation. As alertness originates from the information integration of the DMN and other extensive cortical networks, the thalamus provides a platform for information integration. ${ }^{40}$ The thalamus is closely related with loss of consciousness, while the changes of consciousness result from complex interactions between the DMN-based cortical network, thalamus, and brain stem. ${ }^{41}$ The thalamus acts as a swift conversion factor from the presence to loss of alertness, ${ }^{41}$ while the DMN presents a better correlation with gradual changes of alertness caused by anesthetics. ${ }^{10,22}$ The thalamus is closely correlated with loss of consciousness and its inhibition requires a larger dose of anesthetics. ${ }^{42}$ Previous studies have revealed the role of corticothalamic interactions in the maintenance of alertness and the effect of propofol on these interactions, ${ }^{43,44}$ which support the present study. The present study suggests that there is no change in connectivity between the mediodorsal thalamus and the DMN under sedation. Nevertheless, there are some discrepancies between the present study and these previous studies, but they may be due to the methodology, the patients, and the fact that we did not achieve loss of alertness.

This study has some limitations. Our experiment did not include a loss of consciousness state, since loss of consciousness with increasing propofol dose is often accompanied by respiratory depression, leading to elevation of end-tidal carbon dioxide pressure that may affect the accuracy of the data. In addition, prolonged experimental duration may irritate the participants and affect the experimental results.

\section{Conclusion}

The DMN is the key target in sedation and hypnosis using propofol and it is gradually inhibited with decreasing level of alertness. The overall DMN is sensitive to propofol, in which it can be significantly inhibited by a small dose of propofol. PCC, aMPFC, and LatP are less sensitive to propofol, and they are significantly inhibited by a high concentration of propofol, further reducing the level of alertness.

\section{Acknowledgment}

The authors acknowledge Tianzai Jiang (Brainnetome Center, Institute of Automation, Chinese Academy of Sciences, Beijing, People's Republic of China) for his help in study design and data processing.

\section{Disclosure}

The authors report no conflicts of interest in this work.

\section{References}

1. Desai M, Kahn I, Knoblich U, et al. Mapping brain networks in awake mice using combined optical neural control and fMRI. J Neurophysiol. 2011;105(3):1393-1405.

2. Raichle ME, MacLeod AM, Snyder AZ, Powers WJ, Gusnard DA, Shulman GL. A default mode of brain function. Proc Natl Acad Sci USA. 2001;98(2):676-682.

3. Sämann PG, Wehrle R, Hoehn D, et al. Development of the brain's default mode network from wakefulness to slow wave sleep. Cereb Cortex. 2011;21(9):2082-2093.

4. Fernández-Espejo D, Soddu A, Cruse D, et al. A role for the default mode network in the bases of disorders of consciousness. Ann Neurol. 2012;72(3):335-343.

5. Buckner RL, Andrews-Hanna JR, Schacter DL. The brain's default network: anatomy, function, and relevance to disease. Ann N Y Acad Sci. 2008;1124:1-38

6. Cabeza R, Dolcos F, Graham R, Nyberg L. Similarities and differences in the neural correlates of episodic memory retrieval and working memory. Neuroimage. 2002;16(2):317-330.

7. Toussaint PJ, Maiz S, Coynel D, et al. Characteristics of the default mode functional connectivity in normal ageing and Alzheimer's disease using resting state fMRI with a combined approach of entropy-based and graph theoretical measurements. Neuroimage. 2014;101:778-786.

8. Yao N, Shek-Kwan Chang R, Cheung C, et al. The default mode network is disrupted in Parkinson's disease with visual hallucinations. Hum Brain Mapp. 2014;35(11):5658-5666.

9. Deshpande G, Kerssens C, Sebel PS, Hu X. Altered local coherence in the default mode network due to sevoflurane anesthesia. Brain Res. 2010; 1318:110-121.

10. Schrouff J, Perlbarg V, Boly M, et al. Brain functional integration decreases during propofol-induced loss of consciousness. Neuroimage. 2011;57(1):198-205.

11. Stamatakis EA, Adapa RM, Absalom AR, Menon DK. Changes in resting neural connectivity during propofol sedation. PLoS One. 2010; 5(12): 14224

12. Guldenmund P, Demertzi A, Boveroux P, et al. Thalamus, brainstem and salience network connectivity changes during propofol-induced sedation and unconsciousness. Brain Connect. 2013;3(3):273-285.

13. Gili T, Saxena N, Diukova A, Murphy K, Hall JE, Wise RG. The thalamus and brainstem act as key hubs in alterations of human brain network connectivity induced by mild propofol sedation. J Neurosci. 2013;33(9):4024-4031.

14. Miller RD, Eriksson LI, Fleisher LA, Wiener-Kronish JP, Young WL, editors. Miller's Anesthesia. 7th Edition. New York: Churchill Livingstone; 2010.

15. Hernández-Gancedo C, Pestaña D, Peña N, Royo C, PérezChrzanowska $\mathrm{H}$, Criado A. Monitoring sedation in critically ill patients: bispectral index, Ramsay and observer scales. Eur J Anaesthesiol. 2006; 23(8):649-653.

16. Chao-Gan Y, Yu-Feng Z. DPARSF: A MATLAB Toolbox for "Pipeline" Data Analysis of Resting-State fMRI. Front Syst Neurosci. 2010;4:13.

17. Fair DA, Cohen AL, Dosenbach NU, et al. The maturing architecture of the brain's default network. Proc Natl Acad Sci U S A. 2008; 105(10):4028-4032.

18. Song $\mathrm{M}, \mathrm{Du} \mathrm{H}, \mathrm{Wu} \mathrm{N}$, et al. Impaired resting-state functional integrations within default mode network of generalized tonic-clonic seizures epilepsy. PLoS One. 2011;6(2):e17294.

19. Onnela JP, Saramäki J, Kertész J, Kaski K. Intensity and coherence of motifs in weighted complex networks. Phys Rev E Stat Nonlin Soft Matter Phys. 2005;71(6 Pt 2):065103.

20. Rubinov M, Sporns O. Complex network measures of brain connectivity: uses and interpretations. Neuroimage. 2010;52(3):1059-1069.

21. Achard S, Bullmore E. Efficiency and cost of economical brain functional networks. PLoS Comput Biol. 2007;3(2):e17.

22. Boveroux P, Vanhaudenhuyse A, Bruno MA, et al. Breakdown of within- and between-network resting state functional magnetic resonance imaging connectivity during propofol-induced loss of consciousness. Anesthesiology. 2010;113(5):1038-1053. 
23. Greicius MD, Krasnow B, Reiss AL, Menon V. Functional connectivity in the resting brain: a network analysis of the default mode hypothesis. Proc Natl Acad Sci U S A. 2003;100(1):253-258.

24. Andrews-Hanna JR, Reidler JS, Sepulcre J, Poulin R, Buckner RL. Functional-anatomic fractionation of the brain's default network. Neuron. 2010;65(4):550-562.

25. Mantini D, Gerits A, Nelissen K, et al. Default mode of brain function in monkeys. J Neurosci. 2011;31(36):12954-12962.

26. Fransson $P$, Marrelec $G$. The precuneus/posterior cingulate cortex plays a pivotal role in the default mode network: Evidence from a partial correlation network analysis. Neuroimage. 2008;42(3):1178-1184.

27. Greicius MD, Kiviniemi V, Tervonen O, et al. Persistent default-mode network connectivity during light sedation. Hum Brain Mapp. 2008; 29(7):839-847.

28. D'Argembeau A, Ruby P, Collette F, et al. Distinct regions of the medial prefrontal cortex are associated with self-referential processing and perspective taking. J Cogn Neurosci. 2007;19(6):935-944.

29. Mason MF, Norton MI, Van Horn JD, Wegner DM, Grafton ST, Macrae CN. Wandering minds: the default network and stimulusindependent thought. Science. 2007;315(5810):393-395.

30. Schacter DL, Addis DR, Buckner RL. Remembering the past to imagine the future: the prospective brain. Nat Rev Neurosci. 2007;8(9): 657-661.

31. Bar M. The proactive brain: memory for predictions. Philos Trans $R$ Soc Lond B Biol Sci. 2009;364(1521):1235-1243.

32. Vincent JL, Patel GH, Fox MD, et al. Intrinsic functional architecture in the anaesthetized monkey brain. Nature. 2007;447:83-86.

33. Gorges M, Müller HP, Lulé D, Ludolph AC, Pinkhardt EH, Kassubek J. Functional connectivity within the default mode network is associated with saccadic accuracy in Parkinson's disease: a resting-state FMRI and videooculographic study. Brain Connect. 2013;3(3):265-272.

34. Takano K, Ora H, Sekihara K, Iwaki S, Kansaku K. Coherent Activity in Bilateral Parieto-Occipital Cortices during P300-BCI Operation. Front Neurol. 2014;5:74.

35. Lee U, Kim S, Noh GJ, Choi BM, Hwang E, Mashour GA. The directionality and functional organization of frontoparietal connectivity during consciousness and anesthesia in humans. Conscious Cogn. 2009; 18(4):1069-1078.
36. Ono Y, Nanjo T, Ishiyama A. Pursuing the flow of information: connectivity between bilateral premotor cortices predicts better accuracy in the phonological working memory task. Conf Proc IEEE Eng Med Biol Soc. 2013;2013:7404-7407.

37. Dueck MH, Petzke F, Gerbershagen HJ, et al. Propofol attenuates responses of the auditory cortex to acoustic stimulation in a dosedependent manner: a FMRI study. Acta Anaesthesiol Scand. 2005;49(6): 784-791.

38. Plourde G, Belin P, Chartrand D, et al. Cortical processing of complex auditory stimuli during alterations of consciousness with the general anesthetic propofol. Anesthesiology. 2006;104(3):448-457.

39. Tononi G. An information integration theory of consciousness. BMC Neurosci. 2004;5:42.

40. Guillery RW, Sherman SM. Thalamic relay functions and their role in corticocortical communication: generalizations from the visual system. Neuron. 2002;33(2):163-175.

41. Alkire MT, Haier RJ, Fallon JH. Toward a unified theory of narcosis: brain imaging evidence for a thalamocortical switch as the neurophysiologic basis of anesthetic-induced unconsciousness. Conscious Cogn. 2000;9(3):370-386.

42. Heinke W, Koelsch S. The effects of anesthetics on brain activity and cognitive function. Curr Opin Anaesthesiol. 2005;18(6):625-631.

43. Boly M, Moran R, Murphy M, et al. Connectivity changes underlying spectral EEG changes during propofol-induced loss of consciousness. J Neurosci. 2012;32(20):7082-7090.

44. Mhuircheartaigh RN, Rosenorn-Lanng D, Wise R, Jbabdi S, Rogers R, Tracey I. Cortical and subcortical connectivity changes during decreasing levels of consciousness in humans: a functional magnetic resonance imaging study using propofol. J Neurosci. 2010;30(27):9095-9102.
Neuropsychiatric Disease and Treatment

\section{Publish your work in this journal}

Neuropsychiatric Disease and Treatment is an international, peerreviewed journal of clinical therapeutics and pharmacology focusing on concise rapid reporting of clinical or pre-clinical studies on a range of neuropsychiatric and neurological disorders. This journal is indexed on PubMed Central, the 'PsycINFO' database and CAS,

\section{Dovepress}

and is the official journal of The International Neuropsychiatric Association (INA). The manuscript management system is completely online and includes a very quick and fair peer-review system, which is all easy to use. Visit http://www.dovepress.com/testimonials.php to read real quotes from published authors. 